\title{
¿El ecocardiograma transesofágico Doppler color 3D es mejor que el 2D para medir defectos únicos del tabique interauricular en la sala de hemodinamia?
}

\author{
Is the 3D transesophageal Doppler color echocardiogram better than 2D to measure \\ single defects of the interatrial septum in the catheterization laboratory?
}

\author{
Alejandro E. Contreras ${ }^{1 *}$, Adolfo Ferrero-Guadagnoli ${ }^{2}$, Gabriela Vilte ${ }^{1}$ y Alejandro Peirone ${ }^{2}$ \\ ${ }^{1}$ Servicio de Cardiología; ${ }^{2}$ Servicio de Hemodinamia, Hospital Privado Universitario de Córdoba. Córdoba, Argentina.
}

La relación de una comunicación interauricular $(\mathrm{CIA})$ con estructuras adyacentes, la medición de sus dimensiones y la presencia de bordes adecuados son variables fundamentales a la hora de decidir la factibilidad de proponer un cierre por cateterismo ${ }^{1}$. La medición del defecto con balón es un método que ha demostrado ser útil para la elección del tamaño del dispositivo a implantar, usualmente se selecciona un dispositivo de 1 a $2 \mathrm{~mm}$ mayor al tamaño de la cintura evocada por el balón ${ }^{2,3}$. Como soporte y guía, en la sala de hemodinamia es frecuente usar la ecocardiografía transesofágica (ETE) en sus diferentes modalidades (2D-3D). El motivo de nuestro trabajo fue determinar si la medición de la CIA por eco 3D se correlaciona mejor con las medidas por balón que las determinadas con eco 2D.

Realizamos un estudio retrospectivo de los pacientes que fueron intervenidos para cierre percutáneo de CIA entre septiembre de 2015 y marzo de 2018. Incluimos los casos en donde se utilizó eco 3D dentro de la sala de hemodinamia como soporte de imágenes. Correlacionamos la medición del eco 3D y del eco 2D con la medida del balón como estándar de oro. Usamos el Test de correlación de Spearman. Se realizó un grafico de Bland y Altman para evaluar la concordancia entre las dimensiones en ETE 2D y 3D. Se usó el paquete estadístico SPSS 17 y MedCalc 18.11.

Incluimos 12 pacientes (7 varones), con edad promedio de 16.1 años (5-70 años), peso de $40.8 \mathrm{~kg}$ (18-110 kg). Tenían CIA únicas 10 pacientes y múltiples 2 pacientes, el $41.7 \%$ (5 pacientes) tenían borde aórtico deficiente y el $16.7 \%$ (2 pacientes) tenían borde posterior flácido. El diámetro mayor medido por ETE 2D fue $15.4 \mathrm{~mm}$ (9-31 mm), el diámetro mayor por ETE 3D fue $14.9 \mathrm{~mm}(8-31 \mathrm{~mm})$, el diámetro medido por balón fue $17.7 \mathrm{~mm}$ (11-25 mm). Los dispositivos colocados fueron en promedio de $20.5 \mathrm{~mm}$ (14-30 mm). La correlación entre ETE 2D y balón fue buena $(r=0.88 ; p 0.001)$, también entre ETE 3D y balón ( $r=0.83 ; p=0.001)$, así como la concordancia entre ETE 2D y 3D (Figs. 1 y 2).

EI ETE 3D en la sala de hemodinamia es de suma utilidad en la evaluación de defectos múltiples y cierre de defectos residuales ${ }^{4}$. Cerca del $8 \%$ de las comunicaciones interauriculares tipo ostium secundum son múltiples $^{5}$. Se han propuesto fórmulas basadas en ETE 3D para la elección del tamaño del dispositivo a implantar ${ }^{6}$, así como la utilidad del 3D para evitar el uso de balón en la medición del defecto ${ }^{7}$, sin embargo,

\section{Correspondencia:}

*Alejandro E. Contreras

Naciones Unidas, 346

Barrio Parque Vélez Sársfield

Fecha de recepción: 15-06-2018

C.P. X5016KEH, Córdoba, Argentina Fecha de publicación: 10-12-2018

E-mail: aletreras@ @otmail.com

DOI: 10.24875/ACM.M19000038

Disponible en internet: 06-05-2019 Arch Cardiol Mex. 2019;89(3):270-272 www.archivoscardiologia.com 1405-9940 @ 2018 Instituto Nacional de Cardiología Ignacio Chávez. Publicado por Permanyer México SA de CV. Este es un artículo Open Access bajo la licencia CC BY-NC-ND (http://creativecommons.org/licenses/by-nc-nd/4.0/). 


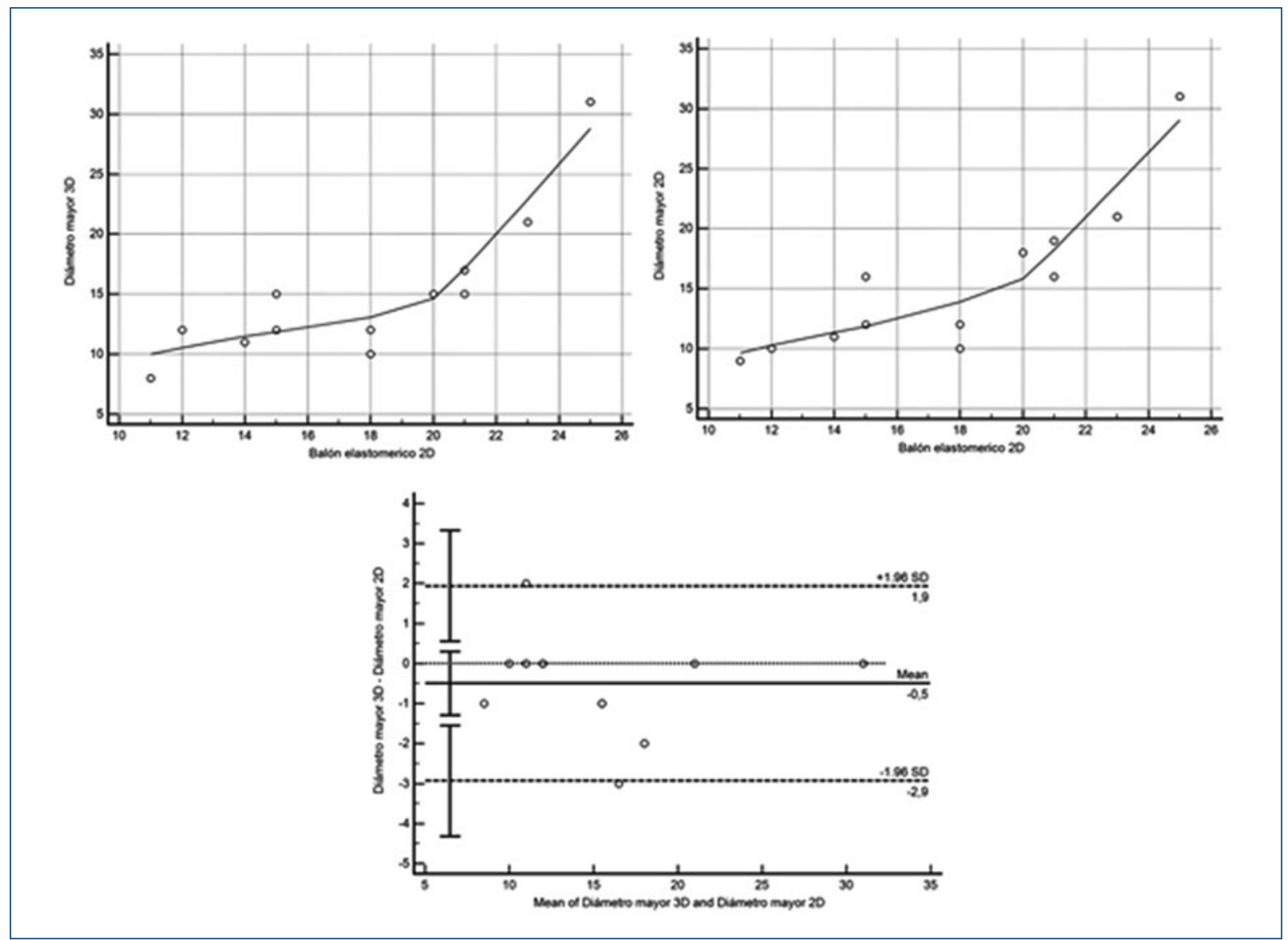

Figura 1. Correlación entre eco 3D y balón (izquierda, $r=0.83$ ) y entre eco $2 \mathrm{D}$ y balón (centro, $r=0.88$ ). Gráfico de Bland y Altman que muestra la concordancia entre diámetro mayor por eco $3 \mathrm{D}$ y $2 \mathrm{D}(\mathrm{p}=0.19)$.

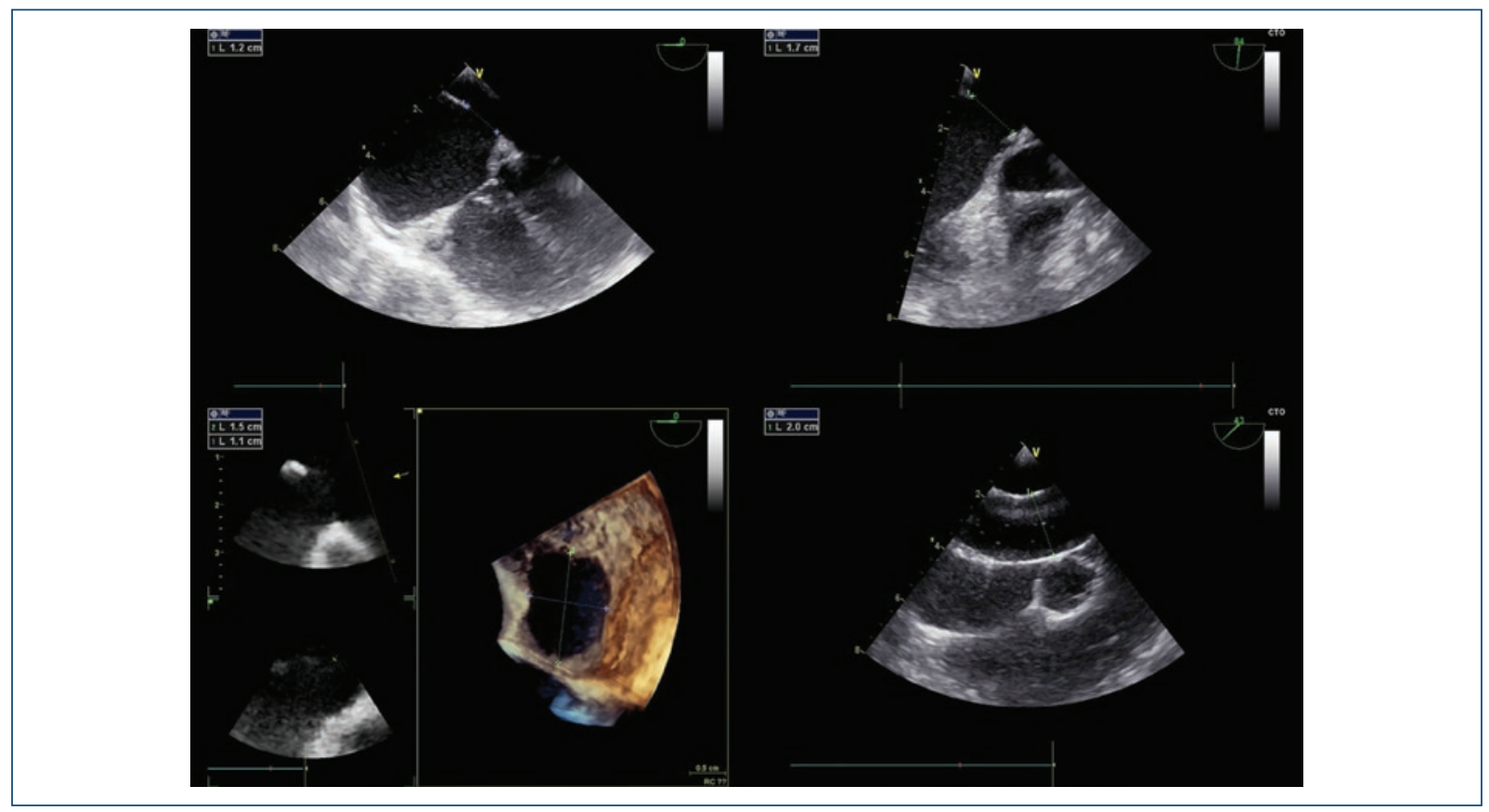

Figura 2. Medición en ecocardiograma transesofagico bidimensional (superior izquierda y derecha, 0 y 90 grados, respectivamente). Medición en ecocardiograma transesofagico tridimensional (abajo izquierda). Medición con balón (abajo derecha). 
ningún estudio ha demostrado que el ETE 3D sea claramente mejor en la medición de defectos únicos ${ }^{4,6,8}$. En nuestra experiencia, ambos métodos (ETE 2D y 3D) tuvieron buena correlación con la medición con balón, pero no hubo diferencias entre el ETE 2D y 3D.

Las imágenes tridimensionales son esenciales para el hemodinamista. Mejoran la orientación espacial en algunas circunstancias, como por ejemplo en el momento del implante de mitraclip o con defectos múltiples del septum interauricular. La principal desventaja del 3D es la restricción en cuanto al peso mínimo requerido de los pacientes, ya que no existen sondas pediátricas y se recomienda su uso en pacientes mayores de $35 \mathrm{~kg}$, aunque, en nuestro caso, en un paciente con $18 \mathrm{~kg}$ se pudo usar sin complicaciones.

En la mayoría de las comunicaciones interauriculares tipo ostium secundum, el ETE 2D es el método de elección para el control en la sala de hemodinamia ${ }^{5}$, especialmente si el septum interauricular no es flácido; en cambio, si el defecto es mas complejo, oval o múltiple, el ETE 3D puede aportar una ventaja en la evaluación anatómica y para la elección de dispositivos ${ }^{9}$.

\section{Conflicto de intereses}

Los Dres. Contreras, Ferrero Guadagnoli y Vilte no poseen conflictos de interés. El Dr. Peirone es Consultor y Proctor de pfm Medical, Proctor de St Jude Medical, Proctor de Occlutech Medical y Proctor de Cera Lifetech Science.

\section{Financiamiento}

Ninguno.

\section{Responsabilidades éticas}

Protección de personas y animales. Los autores declaran que para esta investigación no se han realizado experimentos en seres humanos ni en animales.

Confidencialidad de los datos. Los autores declaran que han seguido los protocolos de su centro de trabajo sobre la publicación de datos de pacientes.

Derecho a la privacidad y consentimiento informado. Los autores han obtenido el consentimiento informado de los pacientes y/o sujetos referidos en el artículo. Este documento obra en poder del autor de correspondencia.

\section{Bibliografía}

1. Mathewson JW, Bichell D, Rothman A, Ing FF. Absent posteroinferior and anterosuperior atrial septal defect rims: Factors affecting nonsurgical closure of large secundum defects using the Amplatzer occluder. J Am Soc Echocardiogr. 2004;17:62-9.

2. Du ZD, Cao Q, Rhodes J, Heitschmidt M, Hijazi ZM. Choice of size and results of transcatheter closure of atrial septal defect using the Amplatzer septal occluder. J Interv Cardiol. 2002;15:287-92.

3. Harper RW, Mottram PM, Mc Gaw DJ. Closure of secundum atrial septal defects with the Amplatzer septal occluder device: Techniques and problems. Catheter Cardiovasc Interv. 2002:57:508-24.

4. Simpson J, Lopez L, Acar P, Friedberg MK, Khoo NS, Ko HH, et al. Three dimensional echocardiography in congenital heart disease: An expert consensus document from the European Association of Cardiovascular Imaging and the American Society of Echcardiography. J Am Soc Echocardiogr. 2017;30:1-27

5. Sobrino A, Basmadjian AJ, Ducharme A, Ibrahim R, Mercier LA Pelletier $\mathrm{GB}$ et al. Ecocardiografía transesofagica multiplanar para la evaluación y el tratamiento percutáneo de la comunicación interauricular tipo ostium secundum en el adulto. Arch Cardiol Mex. 2012;82:37-47.

6. Jang JY, Heo R, Cho MS, Bae J, Hong JA, Lee S, et al. Efficacy of 3D transoesophageal echocardiography for transcatheter device closure of atrial septal defect without ballon sizing. Eur Heart J Cardiovasc Imaging. 2018;19:684-9.

7. Hascoet S, Hadeed K, Marchal P, Dulac Y, Alacoque X, Heitz T, et al The relation between atrial septal defect shape, diameter, and area using three-dimensional transoesophageal echocardiography and balloon sizing during percutaneous closure in children. Eur Heart $\mathrm{J}$ Cardiovasc Imaging. 2015;16:747-55

8. Bhaya M, Mutluer FO, Mahan E, Mahan L, Hsiung MC, Yin WH, et al. Live/ real time three-dimensional transoesophageal echocardiography in percutaneous closure of atrial septal defects. Echocardiography. 2013:30:345-53.

9. Boon I, Vertongen K, Paelinck BP, Demulier L, Berendoncks AV, De Maeyer $\mathrm{C}$, et al. How to size ASDs for percutaneous closure. Pediatr Cardiol. 2018;39:168-75 